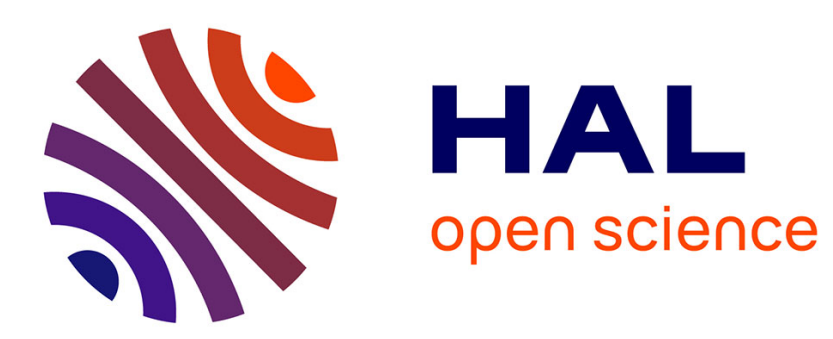

\title{
INVESTIGATION AND MODELING OF DAMPING IN A PLATE WITH A BONDED POROUS LAYER
}

\author{
Nicolas Dauchez, Sohbi Sahraoui, Noureddine Atalla
}

\section{To cite this version:}

Nicolas Dauchez, Sohbi Sahraoui, Noureddine Atalla. INVESTIGATION AND MODELING OF DAMPING IN A PLATE WITH A BONDED POROUS LAYER. Journal of Sound and Vibration, 2003, 265 (2), pp.437-449. hal-00438860

\section{HAL Id: hal-00438860 https://hal.science/hal-00438860}

Submitted on 31 Dec 2009

HAL is a multi-disciplinary open access archive for the deposit and dissemination of scientific research documents, whether they are published or not. The documents may come from teaching and research institutions in France or abroad, or from public or private research centers.
L'archive ouverte pluridisciplinaire HAL, est destinée au dépôt et à la diffusion de documents scientifiques de niveau recherche, publiés ou non, émanant des établissements d'enseignement et de recherche français ou étrangers, des laboratoires publics ou privés. 


\title{
INVESTIGATION AND MODELING OF DAMPING IN A
} PLATE WITH A BONDED POROUS LAYER

\author{
Nicolas Dauchez and Sohbi SAhraoui \\ Laboratoire d'Acoustique UMR CNRS 6613, Univ. du Maine, \\ 72085 Le Mans cedex 9, France \\ Noureddine ATALla \\ GAUS, Mechanical Engineering Dpt, Université de Sherbrooke, \\ Sherbrooke, Québec, J1K 2R1, Canada
}




\section{SUMMARY}

Behavior of a poroelastic material bonded onto a vibrating plate is investigated in the low frequency range. From the analysis of dissipation mechanisms, a model accounting for damping added by the porous layer on the plate is derived. This analysis is based on a 3-D finite element formulation including poroelastic elements based on Biot displacement theory. First, dissipated powers related to thermal, viscous and viscoelastic dissipation are explicited. Then a generic configuration (simply supported aluminium plate with a bonded porous layer and mechanical excitation) is studied. Thermal dissipation is found negligible. Viscous dissipation can be optimized as a function of air flow resistivity. It can be the major phenomenon within soft materials, but for most foams viscoelastic dissipation is dominant. Consequently an equivalent plate model is proposed. It includes shear in the porous layer and only viscoelasticity of the skeleton. Excellent agreement is found with the full numerical model. 


\section{INTRODUCTION}

Porous materials like polymer foam and glass wool are widely used for noise control in several engineering activities such as aeronautics and automotive industries. Their properties are twofold : sound absorption and damping of the nearby structure.

Excited through the coupling with the fluid [1] or directly by the structure when in contact with it [2], the skeleton participate to damping through viscoelastic dissipation.

In the literature, the efficiency of porous material is shown in applications dealing with sound absorption, transmission loss of panels $[3,4]$ or noise in enclosure coupled with elastic panels $[5,6]$. Some are based on finite element calculations including poroelastic elements $[3,5]$. In these studies damping effect is shown for various boundary conditions and materials, but no general tendencies is drawn about the nature of dissipation. Other reports give analytical predictions $[7,8]$ of the importance of viscous dissipation induced by the vibration of the skeleton, but they are approximative and limited to 1-D or 2-D applications.

The present analysis is based on a partition of dissipated and reactive powers in poroelastic media, as similarly proposed by Rasolofosaon [9]. In order to treat any 3-D structure, the calculation relies on a 3-D finite element formulation [10] with poroelastic elements based on Biot displacement theory [11].

Well suited for low frequency range analysis, this formulation has been validated for various applications $[10,12]$. Note that a similar study can be done using the mixed displacement-pressure $(u, P)$ formulation [13]. However, this latter formulation is not used here, since the presented work has been initiated with the $(u, U)$ displacement model. Both formulations have been shown to be equivalent [14]. However the cost of $(u, U)$ formulation is higher since three fluid displacement components are involved while the $(u, P)$ formulation relies on a single pressure degree of freedom for the fluid phase.

A comparison of the different terms of dissipated power gives the relative importance of dissipation mechanisms : viscous, thermal or viscoelastic. The analysis is performed on a structures comprising a simply supported plate associated with a porous layer excited by a point force (figure 1). The influence of its thickness, stiffness and air flow resistivity is studied. The goal is to determine major dissipation mechanisms in order to derive a simplified model of this two layers structrure. This model gives the characteristics of an equivalent plate accounting for mass, stiffness and viscoelastic damping added on the plate. Well suited for low frequency analysis, this model provides an efficient alternative to the full Biot model in terms of reduction of memory requirement and computation time.

\section{POWER PARTITION}

The discretized motion equation of the two layer system is given by Panneton [10]

$$
\left.\left[\begin{array}{cc}
{\left[Z_{e}\right]} & 0 \\
0 & {\left[Z_{p}\right]}
\end{array}\right]\left\{\begin{array}{l}
\{w\} \\
u \\
U
\end{array}\right\}\right\}=\{F\}
$$


where $\left[Z_{e}\right]$ and $\left[Z_{p}\right]$ are respectively the impedance matrix related to the elastic and poroelastic media. $\{F\}$ is the vector of nodal forces, applied to the elastic and poroelastic media. $\{w\},\left\{\begin{array}{l}u \\ U\end{array}\right\}$ are the complex amplitude of the nodal displacements of the plate, the skeleton and the air comprised in the pores respectively. In the case where the porous material is bonded onto the plate, there is a continuity of the displacements at the interface

$$
u=w, \quad U_{n}=u_{n},
$$

where subscript $n$ denotes normal displacement. Tangential fluid displacement is not affected by continuity relations at the interface. These linear relations are applied by multiplication with a contraction matrix.

Harmonic time dependence of the form $e^{j \omega t}$ is assumed so that instantaneous quantities are expressed as

$$
a(t)=\Re\left(a e^{j w t}\right),
$$

where $a$ is complex amplitude and $\Re$ denotes real part. Instantaneous input power $\mathcal{P}(t)$ of the discretized system is the product of the instantaneous velocities by the instantaneous input forces $\mathcal{F}(t)$,

$$
\mathcal{P}(t)=\langle\dot{w}(t), \dot{u}(t), \dot{U}(t)\rangle\{\mathcal{F}(t)\}
$$

where symbols \langle\rangle$,\{\}$ and denote respectively line and column vector, and time derivation. This instantaneous power can be decomposed in two components related to power absorbed by the structure and energy exchanged between the excitation and the structure, such as

$$
\mathcal{P}(t)=D(1+\cos 2(\omega t))+R \sin 2(\omega t) .
$$

The mean power dissipated during one cycle $D$ and the amplitude of the reactive power $R$ derive from the complex power $P$, given by

$$
\begin{aligned}
P & =\frac{1}{2} j \omega\left\langle w^{*}, u^{*}, U^{*}\right\rangle\{F\} \\
& =D+j R,
\end{aligned}
$$

where $^{*}$ denotes a complex conjugate quantity. Using equation (1), complex power can be rewritten in the form

$$
\left.P=\frac{1}{2} j \omega\left\langle w^{*}, u^{*}, U^{*}\right\rangle\left[\begin{array}{cc}
{\left[Z_{e}\right]} & 0 \\
0 & {\left[Z_{p}\right]}
\end{array}\right]\left\{\begin{array}{l}
\{w\} \\
u \\
U
\end{array}\right\}\right\},
$$

depending only on the uncoupled impedance matrix, and on the solution of the global system accounting for interface continuity. For the plate, impedance matrix

$$
\left[Z_{e}\right]=\left[K_{e}\right]-\omega^{2}\left[M_{e}\right],
$$

comprises a real mass matrix $\left[M_{e}\right]$ and a complex stiffness matrix $\left[K_{e}\right]$ accounting for various loss phenomena, such as structural dissipation, acoustic radiation and loss through boundary conditions. Impedance matrix of the poroelastic media is given by Panneton [10]

$$
\left[Z_{p}\right]=\left[K_{p}\right]+j \omega\left[C_{p}\right]-\omega^{2}\left[M_{p}\right] .
$$


This matrix comprises a real mass matrix $\left[M_{p}\right]$, a complex viscous loss matrix $\left[C_{p}\right]$ and a complex stiffness matrix

$$
\left[K_{p}\right]=\left[\begin{array}{ll}
{\left[K_{s s}\right]} & {\left[K_{s f}\right]^{T}} \\
{\left[K_{s f}\right]} & {\left[K_{f f}\right]}
\end{array}\right]
$$

where $\left[K_{s s}\right]$ is the complex stiffness matrix of the solid phase, accounting for structural dissipation. $\left[K_{s f}\right]$ and $\left[K_{f f}\right]$ are complex matrix related respectively to elastic coupling and bulk modulus of the fluid phase. They include also thermal losses from fluid to skeleton. Coupling, viscous and thermal losses are fonction of $\phi$ porosity, $\alpha_{\infty}$ tortuosity, $\sigma$ air flow resistivity, $\Lambda$ and $\Lambda^{\prime}$ viscous and thermal characteristic lengths respectively, according to Johnson-Allard-Lafarge theory $[11,15,16,17]$. Relations between low and high frequency effects are governed by shape factors. Viscous shape factor $M$ is defined by

$$
M=\frac{8 \mu \alpha_{\infty}}{\sigma \phi \Lambda^{2}}
$$

where $\mu$ is the viscosity of the air. Thermal shape factor $M^{\prime}$ is defined by

$$
M^{\prime}=\frac{8 k_{0}^{\prime}}{\phi \Lambda^{2}}
$$

where $k_{0}^{\prime}$ is the thermal permeability. This quantity is deduced from equation 13 assuming $M^{\prime}=1$, as in a cylindrical pore.

Using equations (8-11), complex power can be split into several terms, such as

$$
\begin{aligned}
P= & D_{k e}+D_{k s}+D_{k f}+D_{c p} \\
& +j\left(R_{k e}+R_{k p}+R_{m e}+R_{m p}\right),
\end{aligned}
$$

where $R_{k e}$ and $R_{k p}$ are elastic reactive powers of the plate and porous media. $R_{m e}$ and $R_{m p}$ are inertial reactive powers of the plate and porous media. $D_{k e}, D_{k s}, D_{k f}$ and $D_{c p}$ are dissipated powers related to initial damping of the plate, structural, thermal and 
viscous dissipations within the porous material. Their expressions are

$$
\begin{aligned}
R_{k e}= & \frac{1}{2} \Im\left(j \omega\left\langle w^{*}\right\rangle\left[K_{e}\right]\{w\}\right), \\
R_{k p}= & \frac{1}{2} \Im\left(j \omega\left\langle u^{*}, U^{*}\right\rangle\left[K_{p}\right]\left\{\begin{array}{c}
u \\
U
\end{array}\right\}\right), \\
R_{m e}= & \frac{1}{2} \Im\left(-j \omega^{3}\left\langle w^{*}\right\rangle\left[M_{e}\right]\{w\}\right), \\
R_{m p}= & \frac{1}{2} \Im\left(-j \omega^{3}\left\langle u^{*}, U^{*}\right\rangle\left[M_{p}\right]\left\{\begin{array}{c}
u \\
U
\end{array}\right\}\right. \\
& \left.-j \omega^{2}\left\langle u^{*}, U^{*}\right\rangle\left[C_{p}\right]\left\{\begin{array}{c}
u \\
U
\end{array}\right\}\right), \\
D_{k e}= & \frac{1}{2} \Re\left(j \omega\left\langle w^{*}\right\rangle\left[K_{e}\right]\{w\}\right), \\
D_{k s}= & \frac{1}{2} \Re\left(j \omega\left\langle u^{*}\right\rangle\left[K_{s s}\right]\{u\}\right), \\
D_{k f}= & \frac{1}{2} \Re\left(j \omega\left\langle u^{*}, U^{*}\right\rangle\left[\begin{array}{c}
0 \\
\left.\left[K_{s f}\right]\left[K_{s f}\right]_{f f}^{T}\right]
\end{array}\right]\left\{\begin{array}{c}
u \\
U
\end{array}\right\}\right), \\
D_{c p}= & \frac{1}{2} \Re\left(-j \omega^{2}\left\langle u^{*}, U^{*}\right\rangle\left[C_{p}\right]\left\{\begin{array}{c}
u \\
U
\end{array}\right\}\right),
\end{aligned}
$$

where $\Im$ and $\Re$ denotes respectively imaginary and real parts.

A global loss factor $\eta_{g}$ can be calculated for the whole structure. It is expressed as the ratio of total dissipated power over the elastic reactive power of the structure

$$
\eta_{g}=\frac{D_{k e}+\left(D_{k s}+D_{k f}+D_{c p}\right)}{R_{k e}+R_{k p}} .
$$

This expression is consistent with modal loss factor.

\section{DISSIPATION MECHANISMS}

Dissipation mechanisms are studied for a simply supported aluminium plate with a bonded academic porous layer. Continuity of displacement is insured between the plate and the porous layer according to equation (2).

Along the other faces of the porous layer, skeleton and fluid displacements are not constrained : this means that external fluid loading is neglected. This hypothesis, usually assumed for a heavy structure immerged in a light fluid, might have an influence on our conclusions [18-19]. However, plate-foam experimental tests [20] have shown good correlation between experiments and numerical simulations with the above assumption.

The rectangular plate $(22 \mathrm{~cm} \times 28 \mathrm{~cm})$ is meshed by $13 \times 13$ thin shell quadragular elements. Excitation is achieved by a normal point force on the plate, located at (7.7 $\mathrm{cm}, 9.8 \mathrm{~cm}$ ). The porous layer is meshed by $13 \times 13 \times 7$ linear hexahedric elements. This mesh is suitable for the present study, but could be reconsidered for a more critical application due to slow convergence of linear poroelastic elements [21]. 
Calculations performed for various porous materials and thicknesses show that only structural and viscous dissipations are relevant. Thermal dissipation is always found less than $2 \%$ of the total dissipation. This shows that the fluid is not significantly compressed : this is due firstly to the nature of excitation that does not create a straight compression of the fluid in the pore like an acoustical excitation would do, and secondly to the free boundary conditions of the porous layer that do not constrain the fluid in a given volume.

The relative importance of structural and viscous dissipation is now analyzed by varying the related parameters. Structural dissipation induced by the porous layer on the plate is function of the structural loss factor $\eta_{2}$ and the Young's modulus $E_{2}$ of the skeleton. It is studied for two couples $\left(E_{2}, \eta_{2}\right)$, including most of mineral wool or polymer foam characteristics : $\left(E_{2}=60 \mathrm{kPa}, \eta_{2}=0.07\right)$ used for a soft material and $\left(E_{2}=400 \mathrm{kPa}\right.$, $\left.\eta_{2}=0.15\right)$ for a stiff and more dissipative material. The aluminium plate properties are : thickness $h_{1}=1 \mathrm{~mm}$, Young's modulus $E_{1}=69 \mathrm{GPa}$, Poisson ratio $\nu_{1}=0.33$ and density $\rho_{1}=2778 \mathrm{~kg} / \mathrm{m}^{3}$. The loss factor of the plate $\eta_{1}$ is set to 0.01 to account for various dissipation phenomena such as structural damping, acoustic radiation and losses through boundary.

In the low frequency range, viscous dissipation is mainly related to flow resistivity $\sigma$ of the material. Its influence is determined from $10^{3}$ to $10^{8} \mathrm{Nsm}^{-4}$. Characteristic lengths varies with resistivity so that the used material remains realistic : viscous shape factor $M$ is kept constant (Equation 12) and the ratio between thermal and viscous characteristic lengths is fixed to 2.5. The other parameters are fixed and correspond to usual foam or glass wool characteristics : porosity $\phi=0.98$, tortuosity $\alpha_{\infty}=1.3$, skeleton density $\rho_{2}=40 \mathrm{~kg} / \mathrm{m}^{3}$ and Poisson ratio $\nu_{2}=0$. The influence of thickness $h_{2}$ of the porous layer is also investigated, being set to $2 \mathrm{~cm}$ or $5 \mathrm{~cm}$.

Figure 2.a presents viscous dissipation relative to total dissipation, $D_{c p} /\left(D_{k s}+D_{k f}+\right.$ $\left.D_{c p}\right)$, as a function of flow resistivity, for the two thicknesses and materials. It is given for the first bending mode $\left(65 \mathrm{~Hz}<f_{11}<68 \mathrm{~Hz}\right)$ of the structure.

An optimal value of flow resistivity appears for each case. Its location tends to smaller flow resistivity when thickness increases. This is consistent with the calculation of Okuno [8], given for a sealed poroelastic beam undergoing bending deformation

$$
\sigma \simeq \frac{\phi K_{0}}{2.5 h_{2}^{2} f_{i}}
$$

where $K_{0}$ is the bulk modulus of the air and $f$ the frequency. Relative viscous damping increases with the thickness. It reaches a maximum of $84 \%$ for the soft material, being the major dissipating mechanism. However it is limited to $27 \%$ for the stiff material : structural damping is the major dissipating mechanism.

Figure 2.b presents global loss factor of the multilayer, $\eta_{g}$. For very low air flow resistivity, this factor depends only on the structural damping and on the thickness of the porous layer. For a thickness of $2 \mathrm{~cm}$, only stiff polymer foam adds a significant amount of damping. For soft material, damping becomes significant for a thicker layer and only where the viscous dissipation is important : a maximum loss factor of $6 \%$ is then reached with $5 \mathrm{~cm}$. However, according to Okuno [8], the optimized value of air flow resistivity depends on the frequency : it is not suitable for a large frequency range. 
The most reliable way to get damping in the low frequency range is to optimize viscoelastic dissipation with a most dissipative and stiff skeleton. In that case, the amount of added damping is no more dependent on air flow resistivity. Then, air flow resistivity can be then optimized for other purposes such as acoustic absorption.

\section{EQUIVALENT PLATE MODEL}

Viscoelastic dissipation is now assumed to be the major source of damping. In this context, only the skeleton behavior is relevant. The porous layer can be considered as a monophasic viscoelastic media and characteristics of an equivalent plate to the two layer structure can be determined. Development of such an equivalent plate is interesting to reduce computational cost. Moreover, this model can be coupled with an acoustical admittance model [22] when the porous layer is connected to a cavity : in such a case, both acoustical and structural effects of the porous layer, on the cavity and on the elastic structure, are taken into account respectively.

The geometry of th problem is described by figure 3. The two layers are characterized by Young's modulus $E_{1}$ and $E_{2}$, Poisson's ratios $\nu_{1}$ and $\nu_{2}$, densities $\rho_{1}$ and $\rho_{2}$ and thicknesses $h_{1}$ and $h_{2}$. Subscript 1 refers to the plate and 2 to the porous layer.

For pure bending deformation of the structure, the equivalent plate parameters can be calculated from Ross-Kerwin-Ungar (RKU) theory [23] of multilayer plate. Total bending rigidity $D_{12}$ is simply the sum of bending rigidities $D_{1}$ and $D_{2}$ of the two layers related to the neutral fiber of the plate,

$$
D_{12}=D_{1}+D_{2}
$$

where $D_{1}$ and $D_{2}$ are given in the appendix by equations (48) and (49). The equivalent density is given by

$$
\rho_{12}=\frac{\rho_{1} h_{1}+\rho_{2} h_{2}}{h_{1}+h_{2}} .
$$

Equivalent loss factor is given by the ratio of the imaginary and the real part of the total bending rigidity $D_{12}$. However and as expected RKU theory overestimates the equivalent loss factor in comparison with numerical calculation, for a relatively thick layer (figure 4.b). This confirms that porous layer, past a certain thickness, do not exhibit a pure bending deformation.

A more accurate model is achieved by taking into account shear strain in the porous layer (figure 3). Hamilton's principle is used to get the equation of motion of the two layer plate.

Classical thin plate theory is used for the plate. Only calculation related to the porous layer are exposed. For the porous layer, displacement vector $\{u\}^{T}=\langle u, v, w\rangle$ of a particle is assumed to be

$$
\left\{\begin{array}{l}
u(x, y, z, t)=-z \frac{\partial w}{\partial x}-\left(z-z_{1}\right) \psi_{x} \\
v(x, y, z, t)=-z \frac{\partial w}{\partial y}-\left(z-z_{1}\right) \psi_{y} \\
w(x, y, t)=w
\end{array}\right.
$$


where $w$ is the deflection of the plate, $\psi_{x}$ and $\psi_{y}$ deviation angles due to shear strain (figure 3). The components of the strain tensor $\left\{\epsilon_{2}\right\}^{T}=\left\langle\varepsilon_{x}, \varepsilon_{y}, \gamma_{x y}, \gamma_{x z}, \gamma_{y z}\right\rangle$ are

$$
\left\{\begin{array}{l}
\varepsilon_{x}=-z \frac{\partial^{2} w}{\partial x^{2}}-\left(z-z_{1}\right) \frac{\partial \psi_{x}}{\partial x} \\
\varepsilon_{y}=-z \frac{\partial^{2} w}{\partial y^{2}}-\left(z-z_{1}\right) \frac{\partial \psi_{y}}{\partial y} \\
\gamma_{x y}=-2 z \frac{\partial^{2} w}{\partial x \partial y}-\left(z-z_{1}\right)\left(\frac{\partial \psi_{x}}{\partial y}+\frac{\partial \psi_{y}}{\partial x}\right) \\
\gamma_{x z}=-\psi_{x} \\
\gamma_{y z}=-\psi_{y}
\end{array}\right.
$$

Stress-strain relations are given by

$$
\left\{\sigma_{2}\right\}=\left[H_{2}\right]\left\{\epsilon_{2}\right\}
$$

where $\left\{\sigma_{2}\right\}=\left\langle\sigma_{x}, \sigma_{y}, \tau_{x y}, \tau_{x z}, \tau_{y z}\right\rangle$ is the stress tensor and $\left[H_{2}\right]$ is the viscoelastic tensor for a bidimensional isotropic material

$$
\left[H_{2}\right]=\frac{E_{2}}{1-\nu_{2}^{2}}\left[\begin{array}{ccccc}
1 & \nu_{2} & & & \\
\nu_{2} & 1 & & & \\
& & \frac{1-\nu_{2}}{2} & & \\
& & & \frac{1-\nu_{2}}{2} & \\
& & & & \frac{1-\nu_{2}}{2}
\end{array}\right] .
$$

Strain energy V of the multilayer is the sum of bending strain energy of the plate, bending and shear strain energies of the porous layer

$$
\mathrm{V}=\mathrm{V}_{\text {bending }_{1}}+\mathrm{V}_{\text {bending }_{2}}+\mathrm{V}_{\text {shear }_{2}}
$$

where,

$$
\begin{aligned}
& \mathrm{V}_{b 1}=\frac{1}{2} \frac{E_{1}}{1-\nu_{1}^{2}} \int_{S} \int_{-z_{1}}^{z_{1}}\left\langle\varepsilon_{x}, \varepsilon_{y}, \gamma_{x y}\right\rangle\left[\begin{array}{ccc}
1 & \nu_{1} & \\
\nu_{1} & 1 & \\
& & \frac{1-\nu_{1}}{2}
\end{array}\right]\left\{\begin{array}{c}
\varepsilon_{x} \\
\varepsilon_{y} \\
\gamma_{x y}
\end{array}\right\} d x d y d z \\
& \mathrm{~V}_{b 2}=\frac{1}{2} \frac{E_{2}}{1-\nu_{2}^{2}} \int_{S} \int_{z_{1}}^{z_{2}}\left\langle\varepsilon_{x}, \varepsilon_{y}, \gamma_{x y}\right\rangle\left[\begin{array}{ccc}
1 & \nu_{2} & \\
\nu_{2} & 1 & \\
& & \frac{1-\nu_{2}}{2}
\end{array}\right]\left\{\begin{array}{c}
\varepsilon_{x} \\
\varepsilon_{y} \\
\gamma_{x y}
\end{array}\right\} d x d y d z \\
& \mathrm{~V}_{s 2}=\frac{1}{2} \int_{S} \int_{z_{1}}^{z_{2}}\left\langle\gamma_{x z}, \gamma_{y z}\right\rangle\left\{\begin{array}{l}
\tau_{x z} \\
\tau_{y z}
\end{array}\right\} d x d y d z
\end{aligned}
$$

The kinetic energy of the system is given by

$$
\mathrm{T}=\frac{1}{2} \int_{S} m_{12} \dot{w}^{2} \quad d S
$$

with the equivalent density

$$
m_{12}=\rho_{1} h_{1}+\left((1-\phi) \rho_{s}+\phi \rho_{0}\right) h_{2},
$$


where $\phi$ is the porosity, $\rho_{s}$ is the density of the solid comprising the skeleton and $\rho_{0}$ is the density of the air.

Applying Lagrange's equations respectively to each variable, $w, \psi_{x}$ and $\psi_{y}$, and summing the equations related to $\psi_{x}$ and $\psi_{y}$ gives the two equations of motion

$$
\begin{gathered}
D_{1} \Delta \Delta w+D_{2} \Delta \Delta w+D_{4} \Delta \theta+m_{12} \frac{\partial^{2} w}{\partial t^{2}}=0, \\
D_{3} \Delta \theta+D_{4} \Delta \Delta w-C_{2} \theta=0
\end{gathered}
$$

with $\theta=\frac{\partial \psi_{x}}{\partial x}+\frac{\partial \psi_{y}}{\partial y}$ and $\Delta=\frac{\partial^{2}}{\partial x^{2}}+\frac{\partial^{2}}{\partial y^{2}}$, and where $D_{3}$ and $D_{4}$ are bending rigidities given in appendix by equations (50) and (51). For a steady state motion, $w$ and $\theta$ are assumed to be of the form

$$
\begin{gathered}
w(x, y, t)=w_{0} \sin \left(k_{x} x\right) \sin \left(k_{y} y\right) \sin (\omega t), \\
\theta(x, y, t)=\theta_{0} \sin \left(k_{x} x\right) \sin \left(k_{y} y\right) \sin (\omega t),
\end{gathered}
$$

where $k_{x}$ and $k_{y}$ are wavenumbers associated to the directions $x$ and $y$. They are related to the wavenumber $k$ by

$$
k^{2}=k_{x}^{2}+k_{y}^{2}
$$

If the plate is simply supported and $a \times b$ sized, its modes $\left(r_{a}, r_{b}\right)$ correspond to

$$
k_{x}=\pi \frac{r_{a}}{a} \quad \text { and } \quad k_{y}=\pi \frac{r_{b}}{b},
$$

where $r_{a}$ and $r_{b}$ are modal orders along $\mathrm{x}$ and y directions respectively $\left(r_{a}>1, r_{b}>1\right)$. The dispersion equation of the system $(38$ - 39) can then be written in the form

$$
\omega^{2}=k^{4} \frac{D_{12}}{m_{12}} C_{s}\left(k^{2}\right),
$$

where $C_{s}\left(k^{2}\right)$ is the correction factor of the bending rigidity $D_{12}$, accounting for shear, given by

$$
C_{s}\left(k^{2}\right)=1-\frac{D_{4}^{2} k^{2}}{D_{12}\left(D_{3} k^{2}+C_{2}\right)}
$$

As $C_{s}$ is less than 1, shear will lower the resonance frequencies of the system. $C_{s}$ depends on the wavenumber, thus on frequency. For a simply supported plate, $C_{s}$ can be calculated for discrete values of frequency, corresponding to natural frequencies of the plate, given by equations (43) and (44). However $C_{s}$ can be expressed continuously as a function of frequency by solving equation (44) that is a polynomial equation of the third degree on $k^{2}$.

The developed equivalent model and the full numerical model using poroelastic elements are compared in figure 4, in the case described in section 3 where viscoelastic dissipation is dominating. This is also the case where shear is the most important in the porous layer due to its large thickness. The porous layer is of a $5 \mathrm{~cm}$ thick and corresponds to the stiff material $\left(E_{2}=400 \mathrm{kPa}, \eta_{2}=0.15\right)$ with air flow resistivity $\sigma=$ 
$5000 \mathrm{Nsm}^{-4}$. Characteristics of the equivalent plate are first calculated for the given configuration and frequency range. Then they are introduced as properties of a single plate. Equivalent loss factor is given by the ratio of the imaginary and the real part of the corrected bending rigidity $C_{s}\left(k^{2}\right) D_{12}$.

The RKU model is found insufficient for the plate-foam configuration : damping and eigenfrequencies are overestimated. The proposed equivalent model gives a good estimation of mean quadratic velocity of the plate and global loss factor for the first three modes. Comparison has not been performed for higher modes because of the computational cost of the poroelastic finite element method. However it can be seen that the equivalent plate model tends to underestimate damping as frequency increases. One reason is the increase of viscous forces along with frequency. This shows the limitation of the present model to the low frequency range.

\section{CONCLUSION}

Partition of dissipated and reactive powers is presented according to the 3-D formulation coupling elastic and poroelastic elements. Dissipation analysis has been performed in the low frequency range for a two layers structure comprising a porous layer bonded onto a plate. It shows that viscous and viscoelastic dissipation dominate. According to Okuno [8], viscous dissipation can be optimized by choosing a proper flow resistivity : it becomes the major dissipation mechanism within soft materials. However for stiff polymer foams, viscoelastic dissipation in the skeleton is widely dominating. Because viscous dissipation requires tuning of flow resistivity as a function of thickness and frequency, optimizing damping is most efficiently achieved by using the most viscoelastic and stiff porous material. Determination of an optimized flow resistivity is rather connected to sound absorption properties.

Consequently, an equivalent plate model accounting for the effect of the porous layer on the plate has been derived : it includes shear in the porous layer and only viscoelasticity of the skeleton. Good results have been obtained in comparison with the model using poroelastic elements for the three first modes, even for a thick porous layer.

The analysis performed on the two layers structure demonstrated the validity of the method based on power partition. Relying on a 3-D finite element formulation, this approach can be applied to any structure associating acoustic, elastic and poroelastic media. With a best understanding of the behavior of such structures, suitable simplified models can be derived avoiding the use of poroelastic elements, well-known for high computational cost.

\section{ACKNOWLEDGMENTS}

The authors are grateful to PSA Peugeot Citroën and Agence de l'Environnement et de la Maîtrise de l'Energie (Ademe) who have supported this study. 


\section{REFERENCES}

1. T.F. Johansen, J.-F. Allard and B. Brouard 1995 Acta Acoustica 3, 487-491. Finite element method for predicting the acoustical properties of porous samples.

2. T.E. Vigran, L. Kelders, W. Lauriks, M. Dhainaut and T.F. Johansen 1997 ACUSTICA - Acta acustica 83, 1024-1031. Forced response of a sandwich plate with a flexible core described by a Biot-model.

3. R. Panneton and N. Atalla 1996 Journal of the Acoustical Society of America 100, 346-354. Numerical prediction of sound transmission through finite multilayer systems with poroelastic materials.

4. J.S. Bolton, N.M. Shiau and Y.J. Kang 1995 Journal of Sound and Vibration 91, 317-347. Sound transmission through multi-panel structures lined with elastic porous materials

5. $\quad$ N. Atalla and R. Panneton 1996 Noise Control Engineering Journal 44, 235-243. The effects of multilayer sound-absorbing treatments on the noise field inside a plate backed cavity

6. J.S. Mixson and J.F. Wilby 1995 Aeroacoustics of flight vehicles : theory and practice. Harvey H. Hubbard (Acoustical Society of America)

7. M.A. Biot 1964 Transactions of the ASME 31, 194-198. Theory of buckling of a porous slab and its thermoelastic analogy

8. A. Okuno and H.B. Kingsbury 1989 Journal of Applied Mechanics, 56, 535-540. Dynamic modulus of poroelastic materials

9. P. N. J. Rasolofosaon 1991 Journal of the Acoustical Society of America 89, 15321550. Plane acoustic waves in linear viscoelastic porous media : Energy, particle displacement, and physical interpretation

10. R. Panneton and N. Atalla 1997 Journal of the Acoustical Society of America 101, 3287-3298. An efficient finite element scheme for solving the three-dimensional poroelasticity problem in acoustics

11. J.F Allard 1993 Propagation of sound in porous media : modeling sound absorbing materials. Chapman \& Hall, London

12. N. Dauchez, S. Sahraoui and N. Atalla 2000 Internoise 2000, Nice, France, August 30-September 05, 2000. Experimental validation of 3-D poroelastic finite elements based on Biot theory

13. N. Atalla, R. Panneton and P. Debergue 1998 Journal of the Acoustical Society of America 104, 1444-1452. A mixed displacement-pressure formulation for poroelastic materials

14. S. Gorog, R. Panneton and N. Atalla 1997 Journal of Applied Physics 82, 41924196. Mixed displacement-pressure formulation for acoustic anisotropic open porous media

15. D.L. Johnson, Koplik J. and R. Dashen 1987 Journal of FLuid Mechanics 176, 379-402. Theory of dynamic permeability and tortuosity in fluid-saturated porous media 
16. Y. Champoux, J.-F. Allard 1991 Journal of Applied Physics 70, 1975-1979. Dynamic tortuosity and bulk modulus in air-saturated porous media

17. D. Lafarge, Lemarinier P., Allard J.-F. and Tarnow V. 1997 Journal of the Acoustical Society of America 102, 1995-2006. Dynamic compressibility of air in porous structures at audible frequencies

18. P. Debergue, R. Panneton, N. Atalla 1999 Journal of the Acoustical Society of America 106, 2383-2390. Boundary conditions for the weak formulation of the mixed (u,p) poroelasticity problem

19. M. Etchessahar, S. Sahraoui, B. Brouard 2002 Journal of the Acoustical Society of America submitted for publication. Vibrations of poroelastic plates : mixed displacement-pressure modelisation and experiments

20. N. Dauchez 1999 Ph. D. thesis, Université du Maine, France. Etude vibroacoustique des matériaux poreux par éléments finis

21. N. Dauchez, S. Sahraoui, N. Atalla 2001 Journal of the Acoustical Society of America 109, 33-40. Convergence of poroelastic finite element based on Biot displacement formulation

22. C. Lesueur, M.A. Hamdi 1988 Rayonnement acoustique des structures - Vibroacoustique, Interactions fluide-structure. Eyrolles, Paris

23. D. Ross, E.E. Ungar and Jr E.M. Kerwin 1959 Structural Damping, ASME, NewYork, 49-88. Damping of plate flexural vibrations by means of viscoelastic laminate 


\section{APPENDIX}

$$
\begin{gathered}
\mathrm{V}_{b 1}=\frac{D_{1}}{2} \int_{S}\left(\frac{\partial^{2} w}{\partial x^{2}}\right)^{2}+\left(\frac{\partial^{2} w}{\partial y^{2}}\right)^{2}+2 \nu_{1} \frac{\partial^{2} w}{\partial x^{2}} \frac{\partial^{2} w}{\partial y^{2}}+2\left(1-\nu_{1}\right)\left(\frac{\partial^{2} w}{\partial x \partial y}\right)^{2} d S \\
\mathrm{~V}_{b 2}=\frac{1}{2} \int_{S} D_{2}\left[\left(\frac{\partial^{2} w}{\partial x^{2}}\right)^{2}+\left(\frac{\partial^{2} w}{\partial y^{2}}\right)^{2}+2 \nu_{2} \frac{\partial^{2} w}{\partial x^{2}} \frac{\partial^{2} w}{\partial y^{2}}+2\left(1-\nu_{2}\right)\left(\frac{\partial^{2} w}{\partial x \partial y}\right)^{2}\right] \\
+D_{3}\left[\left(\frac{\partial \psi_{x}}{\partial x}\right)^{2}+\left(\frac{\partial \psi_{y}}{\partial y}\right)^{2}+2 \nu_{2} \frac{\partial \psi_{x}}{\partial x} \frac{\partial \psi_{y}}{\partial y}+\frac{1-\nu_{2}}{2}\left(\frac{\partial \psi_{x}}{\partial y}+\frac{\partial \psi_{y}}{\partial x}\right)^{2}\right] \\
+2 D_{4}\left[\left(\frac{\partial^{2} w}{\partial x^{2}} \frac{\partial \psi_{x}}{\partial x}+\frac{\partial^{2} w}{\partial y^{2}} \frac{\partial \psi_{y}}{\partial y}\right)+\nu_{2}\left(\frac{\partial^{2} w}{\partial x^{2}} \frac{\partial \psi_{y}}{\partial y}+\frac{\partial^{2} w}{\partial y^{2}} \frac{\partial \psi_{x}}{\partial x}\right)\right. \\
\left.+\left(1-\nu_{2}\right) \frac{\partial^{2} w}{\partial x \partial y}\left(\frac{\partial \psi_{x}}{\partial y}+\frac{\partial \psi_{y}}{\partial x}\right)\right] d S
\end{gathered}
$$

with the bending rigidities

$$
\begin{aligned}
D_{1}= & \frac{E_{1}}{1-\nu_{1}^{2}} \int_{-z 1}^{z 1} z^{2} d z=\frac{E_{1} h_{1}^{3}}{12\left(1-\nu_{1}^{2}\right)}, \\
D_{2}= & \frac{E_{2}}{1-\nu_{2}^{2}} \int_{z 1}^{z 2} z^{2} d z=\frac{E_{2}}{1-\nu_{2}^{2}} \frac{z_{2}^{3}-z_{1}^{3}}{3}, \\
D_{3}= & \frac{E_{2}}{1-\nu_{2}^{2}} \int_{z 1}^{z 2}\left(z-z_{1}\right)^{2} d z, \\
D_{4}= & \frac{E_{2}}{1-\nu_{2}^{2}} \int_{z 1}^{z 2} z\left(z-z_{1}\right) d z . \\
& \mathrm{V}_{s 2}=\frac{1}{2} C_{2} \int_{S} \psi_{x}^{2}+\psi_{y}^{2} d S,
\end{aligned}
$$

with the shear rigidity

$$
C_{2}=\kappa h_{2} \frac{E_{2}}{2\left(1+\nu_{2}\right)},
$$

and $\kappa$, accounting for the variation of the shear stresses and strains through the thickness, taken to $\frac{5}{6}$. 


\section{Figure captions}

Figure 1. Simply supported plate with a bonded porous layer and mechanical excitation.

Figure 2. Percentage of viscous dissipation relative to total dissipation in the porous layer (a), global loss factor of the multilayer (b), as a function of flow resistivity for two materials : long dashed line,$-- 2 \mathrm{~cm}$ thick stiff material; continuous line,$- 5 \mathrm{~cm}$ thick stiff material; dotted line $\cdots, 2 \mathrm{~cm}$ thick soft material; short dashed line - - -, $5 \mathrm{~cm}$ thick soft material.

Figure 3. Geometry of the two layer plate with shear.

Figure 4. Comparison of equivalent model results with full discretized structure for a $5 \mathrm{~cm}$ thick low resistive $\left(\sigma=5000 \mathrm{Nsm}^{-4}\right)$ and stiff $\left(E_{2}=400 \mathrm{kPa}, \eta_{2}=0.15\right)$ foam layer. (a) Mean quadratic velocity of the plate, (b) Global loss factor of the multilayer : continuous line -, plate with poroelastic elements; dashed line - - -, equivalent plate with pure bending; dotted line $\cdots$, equivalent plate with shear. 
Figure 1.

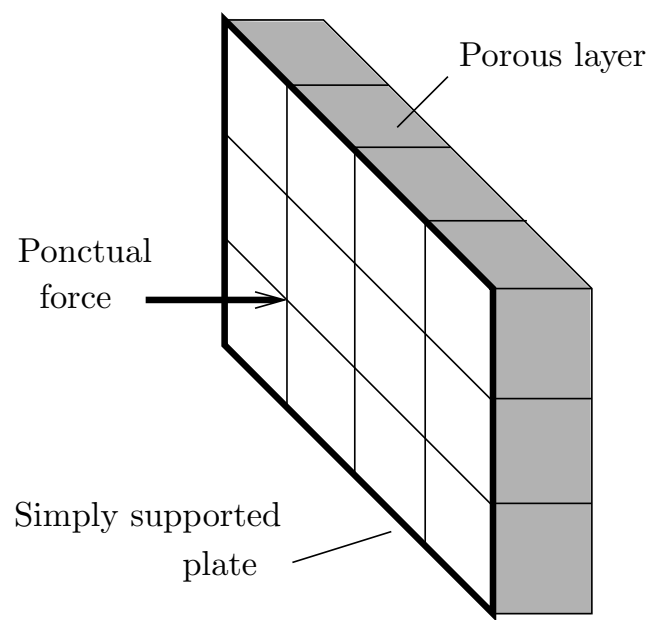


Figure 2.
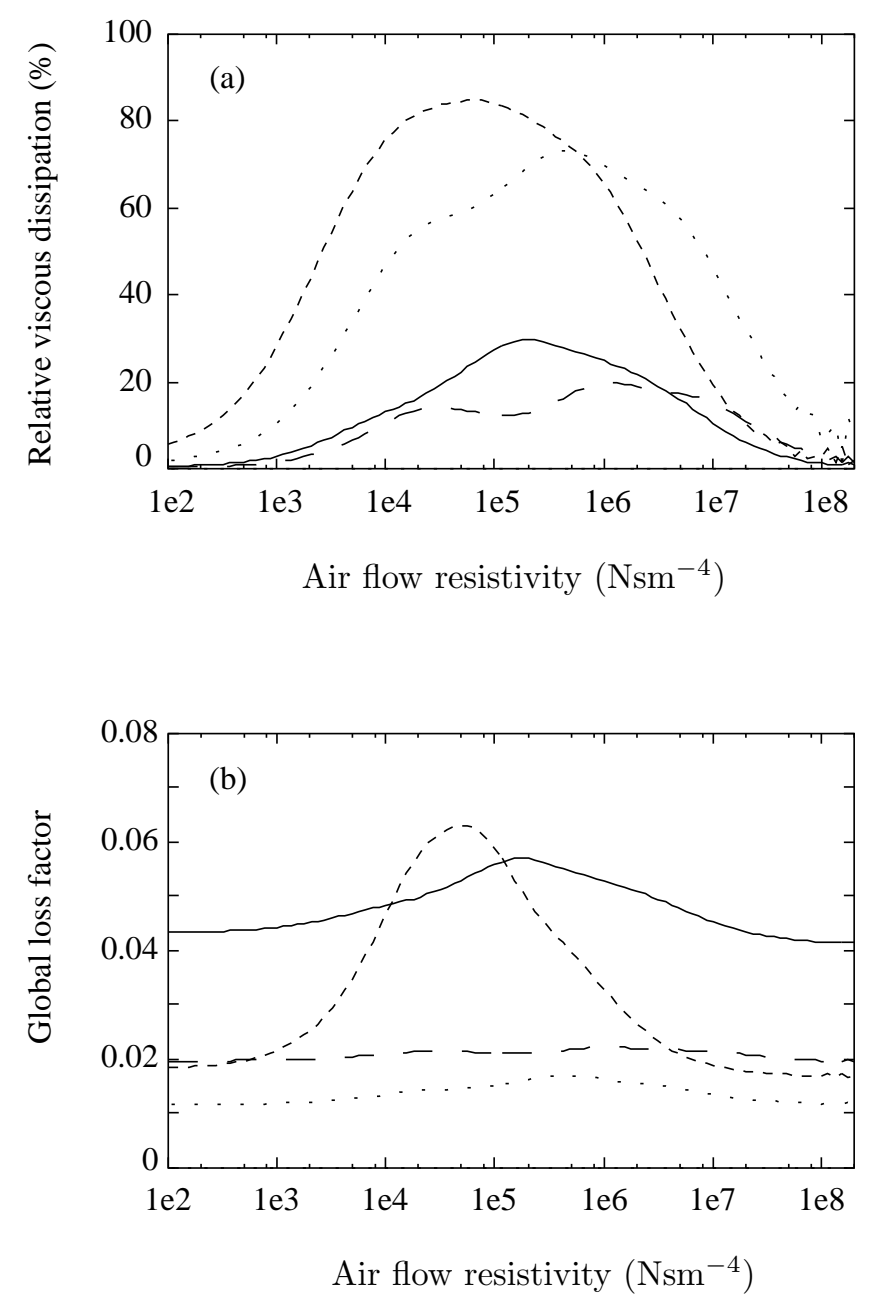
Figure 3.

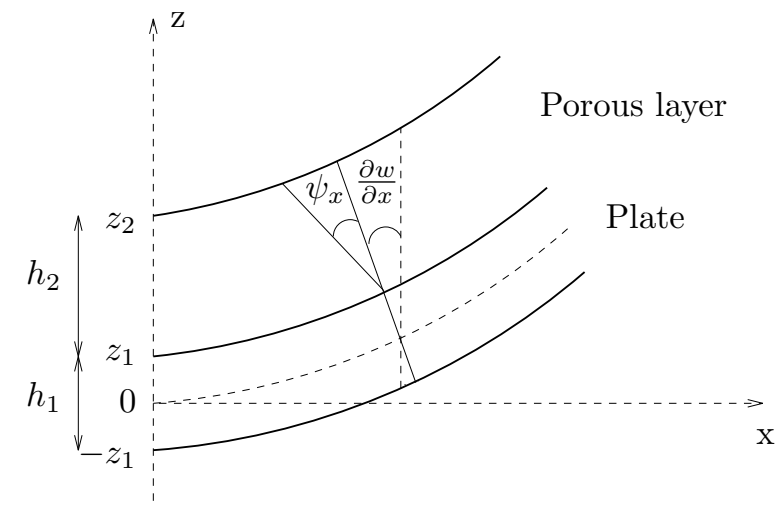


Figure 4.
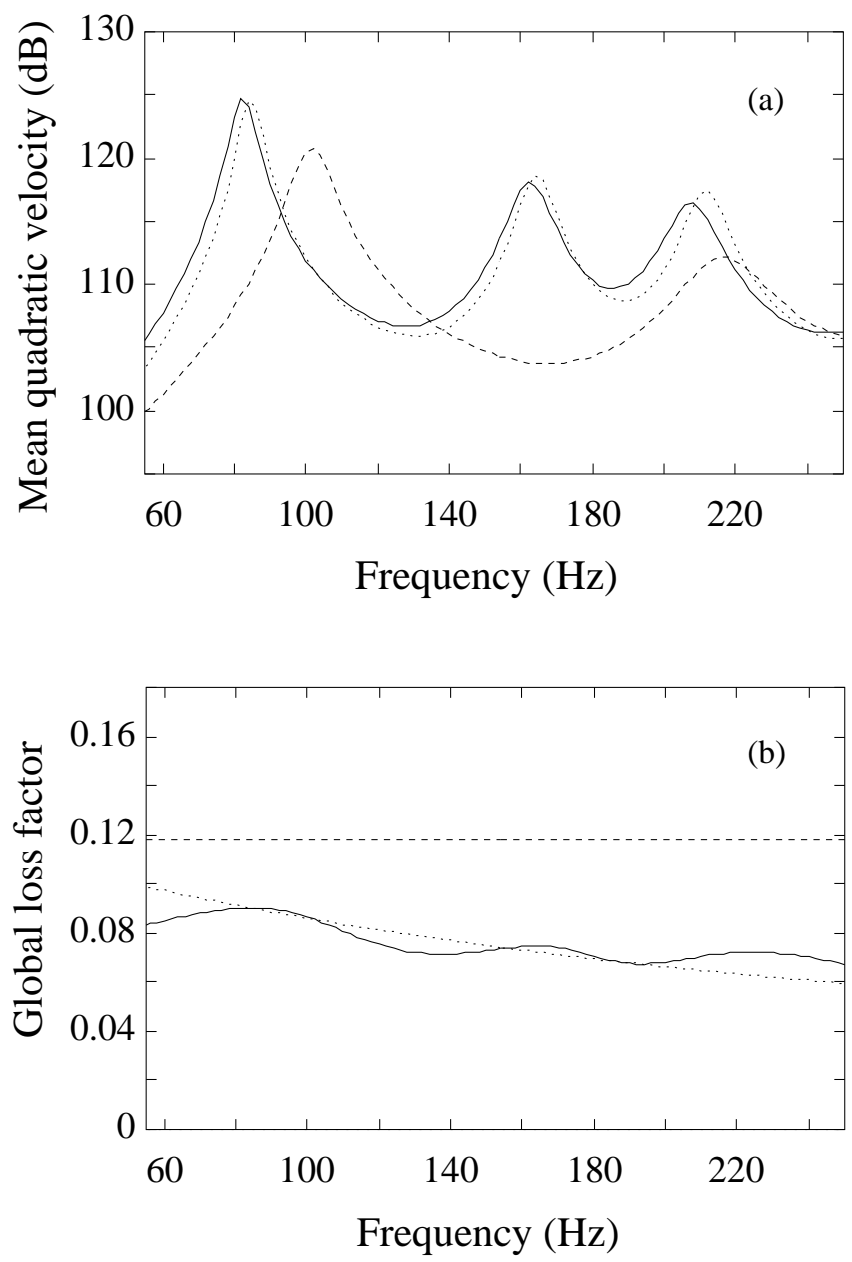